\title{
Effect of Wet Etching of Arc-Induced Long-Period Gratings on Their Refractive Index Sensitivity
}

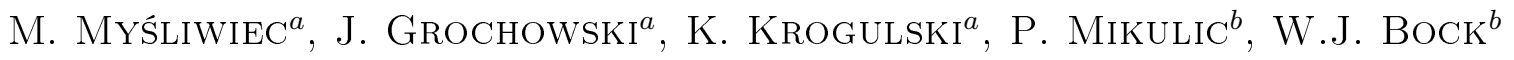 \\ AND M. ŚMIETANA ${ }^{a, *}$ \\ ${ }^{a}$ Institute of Microelectronics and Optoelectronics, Warsaw University of Technology \\ Koszykowa 75, 00-662 Warsaw, Poland \\ ${ }^{b}$ Centre de Recherche en Photonique, Université du Québec en Outaouais, 101 Rue St Jean Bosco \\ Pavillon Lucien Brault, Gatineau, Québec, J8X 3X7, Canada
}

\begin{abstract}
The paper presents highly refractive index sensitive operation of long-period gratings enhanced by wet etching of the fiber cladding. Long-period gratings with period of $283 \mu \mathrm{m}$ were induced in Corning SMF28 optical fibre using electric arc technique. Then etching in hydrofluoric (HF) acid solution has been applied to improve operation of the sensor. Sensitivity improvement effect induced by fiber cladding reduction has been numerically simulated. The developed numerical model enables quick estimation of cladding thickness reduction essential to achieve dispersion turning point. At this point long-period gratings reaches maximum sensitivity to external medium refractive index. Simulation showed that dispersion turning point for this long-period grating is achieved by cladding thickness reduction of $1.7 \mu \mathrm{m}$. We obtained good simulation match with experimental results, where approximately the same thickness was etched. The refractive index sensitivity of the long-period grating-based sensor has been improved by factor of 5 . Since long-period grating period is limited when using electric arc fabrication method, this physical limitation makes difficult achievement of dispersion turning point and reaching maximum sensitivity of the long-period grating. We show that sensitivity of electric arc induced long-period grating can be significantly improved by post processing techniques such as wet etching.
\end{abstract}

DOI: 10.12693/APhysPolA.124.521

PACS: 42.70.Ce, 42.79.Gn, 07.07.Df, 81.20.Fw

\section{Introduction}

There is a great demand of biological and chemical sensors in diagnostics of traces of bio-material in liquids. One of the methods used to characterize such a mixture is measurement of its refractive index (RI). It is known that RI is one of fundamental physical properties of a substance and its measurement has a great potential in the field of biochemistry, pharmaceutics, and biology. Considering these facts sensing device should provide high sensitivity to external medium RI and immediate response. Optical fibre based sensors can easily meet these requirements [1]. Sensors based on long-period gratings (LPGs) are considered a very promising platform for RI sensing. LPGs were reported for the first time by Vensarkar et al. [2]. The device is based on periodical modulation of refractive index of fibres' core. Typical period $(\Lambda)$ of LPG is $140-700 \mu \mathrm{m}$, which is much longer than for the Bragg gratings [3]. This periodic fibre structure causes coupling of guided core mode to series of cladding modes. As a result of the coupling in transmission spectrum of the LPG appear power losses at specific wavelengths $\lambda^{m}$ described by Eq. (1), where $n_{\mathrm{eff}}^{\text {co }}$ is core effective refractive index, $n_{\mathrm{eff}}^{\mathrm{cl} m}$ is effective index of $m$-th order of cladding mode and $\Lambda$ is grating period

*corresponding author; e-mail: M.Smietana@elka.pw.edu.pl

$$
\lambda^{m}=\left(n_{\mathrm{eff}}^{\mathrm{co}}-n_{\mathrm{eff}}^{\mathrm{cl}, m}\right) \Lambda .
$$

RI sensing mechanism is based on the change of $n_{\mathrm{eff}}^{\mathrm{cl}, m}$, when external RI changes. In consequence of the increase of RI the resonant peaks shift towards shorter wavelengths. Dynamics of the shift in observed spectral range depends on cladding mode order, which in turn is determined by proper selection of grating period. Short grating period (typically down to $140 \mu \mathrm{m}$ ) causes higher order cladding mode coupling in wavelength range of about $\lambda=1550 \mathrm{~nm}[4]$. Maximum available sensitivity can be obtained when resonant wavelength is near dispersion turning point (DTP). At this point there are two resonant peaks observed for single cladding mode. Shortly before achieving DTP resonance peaks shift RI change is the highest. Wavelength of DTP is dependent on properties of the fiber and period of LPG [4]. It was proven that DTP is at about $\lambda=1550 \mathrm{~nm}$, when $\Lambda$ is about $200 \mu \mathrm{m}$ [4]. Such short period can be achieved mainly by one of the fabrication methods, which is UV irradiation of the photosensitive fiber.

LPG can be also manufactured using a number of other methods, i.e., $\mathrm{CO}_{2}$ [5] or femtosecond [6] laser irradiation, electric arc discharge [7], periodic etching of the cladding [8], or mechanical stress [9]. The method based on electric arc allows for induction of LPGs in various optical fibres at relatively low cost of the equipment. The main advantage of this process is thermal stability of the gratings [7]. On the other hand, limitation of this method 
is grating fabrication with a short period (depending on a fiber, below $220 \mu \mathrm{m} \mathrm{[10]).} \mathrm{As} \mathrm{mentioned} \mathrm{above,} \mathrm{it} \mathrm{is}$ necessary to make gratings with short period in order to reach the DTP and obtain the highest sensitivity.

In this work we discuss possibility of improving sensitivity of arc-induced LPGs by thinning cladding of the fiber. Up to date in literature there are works on etching of UV induced LPG to tune resonant wavelength to specific point [11]. Authors of this work have shown that it is possible to post-process LPG to shift resonant peak to specific wavelength by etching cladding of the fiber. In this work we apply the method to improve RI sensitivity of arc-induced LPGs and reaching DTP. In the paper we compare numerical simulations and experiment results for thinning fibre cladding and its influence on RI sensitivity of the LPG.

\section{Experimental details}

\section{1. $L P G$ manufacturing}

Single mode fibre Corning SMF28 and electric arc discharge technique based on Fittel S182K Fusion Splicer were used to manufacture the LPGs [10]. Optical fibre was kept at constant tension during LPG fabrication using $2 \mathrm{~g}$ weight [12]. LPGs were written with $282 \mu \mathrm{m}$ period and $57 \mathrm{~mm}$ length. This is shortest period available for achieving good quality LPGs in SMF28 fibre.

\subsection{Simulations}

In order to determine influence of diameter reduction of LPGs on its sensing properties numerical simulations were performed. We used LPG model developed for Optigrating by Optiwave software, which is specially designed for evaluation of LPG performance. The software allows to define fibre as well as grating parameters. The following parameters of the fibre can be determined: core and cladding radii $\left(\rho_{\text {core }}\right.$ and $\left.\rho_{\text {clad }}\right)$, core, cladding and external medium refractive indices $\left(n_{\text {core }}\right.$, $n_{\text {clad }}$ and $\left.n_{\text {ext }}\right)$. Grating parameters can be specified by period $(\Lambda)$, length $(L)$, core refractive index modulation $(\Delta n)$, and grating shape (sine, rectangle). Cladding refractive index and LPG shape were constant for all simulations and set to $n_{\text {clad }}=1.44402$ (refractive index of $\mathrm{SiO}_{2}$ at $1550 \mathrm{~nm}$ ) and LPG shape was assumed to be sinusoid.

\subsection{Wet etching}

Reduction of cladding was performed by wet etching of the fused silica cladding. One of many etchants used in microelectronics technology is HF. In this experiment HF buffer (HF: $\mathrm{NH}_{4} \mathrm{~F}$ 1:40) was used. Chemical reaction between silica glass and $\mathrm{HF}$ etchant is described by formula

$$
\mathrm{SiO}_{2}+6 \mathrm{HF} \rightarrow \mathrm{H}_{2} \mathrm{SiF}_{6}+\mathrm{H}_{2} \mathrm{O}^{-} .
$$

Firstly etch speed was estimated using thermally oxidized silicon wafers. Ellipsometric measurement has been performed before and after 1 min long etching process.
Cladding removal was performed by immersing LPG in etching solution for $60 \mathrm{~s}$. Then fibre was rinsed in deionized water. After that, observation of transmission spectrum was performed to investigate resonance peak shift. Just before achieving DTP, etching was performed in $30 \mathrm{~s}$ intervals for precise resonant wavelength position control.

\subsection{RI sensitivity measurements}

Set of liquids with RI in range from 1.328 to 1.4032 was prepared by mixing water with glycerin in different proportions. Refractive index $\left(n_{\mathrm{D}}\right)$ was measured using Reichert AR200 automatic refractometer. LPG was installed in custom made U-type holder for maintaining constant tension during immersion in liquid. Transmission spectrum was measured in wavelength range from $1100 \mathrm{~nm}$ to $1650 \mathrm{~nm}$. Then LPG was rinsed in deionized water and dried in air. This process was repeated for each RI, before and after fibre etching. Sensitivity was calculated as resonance wavelength shift induced by variation of RI. Sensitivity is defined as ratio of resonance wavelength shift to refractive index unit (RIU):

$$
S_{\mathrm{RI}}=\frac{\Delta \lambda[\mathrm{nm}]}{\Delta n[\mathrm{RIU}]} .
$$

\section{Results and discussion}

\subsection{Simulations of the $L P G$ cladding radius reduction}

Before cladding reduction model of the LPG was adapted to measured spectrum. Some of LPG parameters were known from fabrication process. Parameters of the fabricated LPG and fitted model are shown in Table. Match of the resonant wavelength at $1370 \mathrm{~nm}$ is shown in Fig. 1. The biggest difference in parameters may be observed for LPG length and period. Length of LPG influences only resonance wavelength attenuation, which is not a critical parameter for this work. The electric-arc fabrication method may be responsible for mismatch of LPG period. During fibre processing, equipment works at its limits, which probably causes period disagreement.

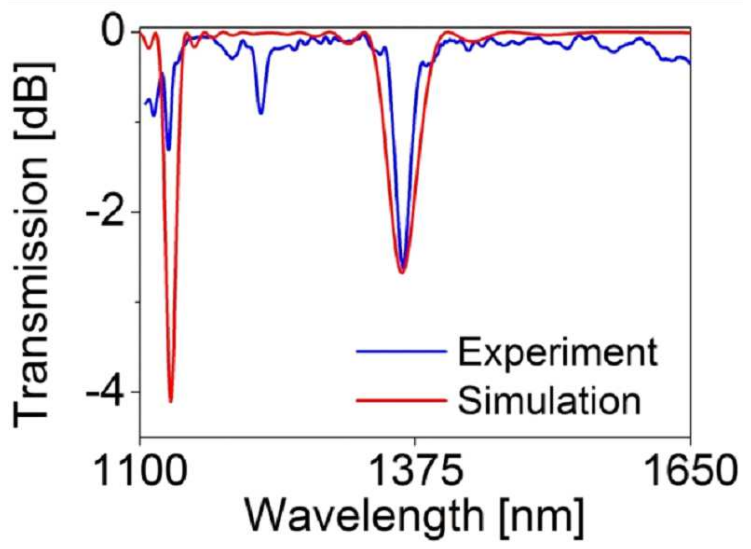

Fig. 1. Transmission spectrum of fabricated LPG and generated using numerical model. 
TABLE

Parameters of fabricated LPG and fitted model. Unknown values marked with "?".

\begin{tabular}{c|c|c}
\hline \hline Parameter & Fabricated LPG & Model \\
\hline$\rho_{\text {core }}[\mu \mathrm{m}]$ & 4.5 & 4 \\
$\rho_{\text {cladd }}[\mu \mathrm{m}]$ & $<58$ & 54.15 \\
$n_{\text {core }}$ & $?$ & 1.44935 \\
$n_{\text {clad }}$ & 1.44402 & 1.44402 \\
period $[\mu \mathrm{m}]$ & 283 & 225 \\
length $[\mathrm{mm}]$ & 57 & 18.5 \\
RI modulation & $?$ & 0.00014
\end{tabular}
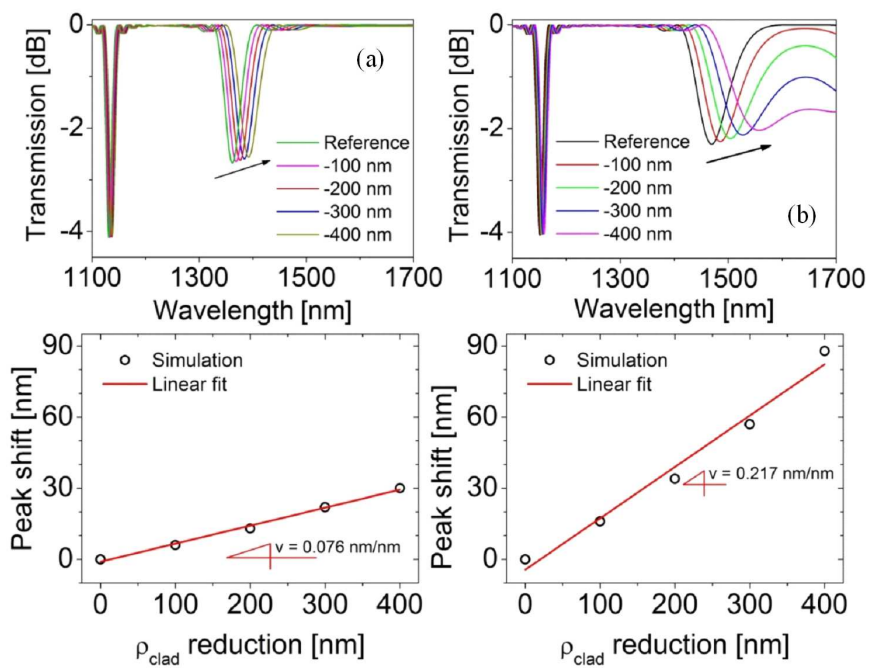

Fig. 2. Simulation results for thinning of the LPG, where (a) shows results for conditions far from DTP and (b) close to DTP. Upper and lower graphs show simulated transmission spectrum and resonant wavelength shift in function of $\rho_{\text {clad }}$ reduction, respectively.

Simulation results of reduction in fiber cladding radius show that far from DTP thinning the fibres' cladding causes $1 \mathrm{~nm}$ resonance wavelength shift on $1 \mathrm{~nm}$ cladding radius reduction of $0.076 \mathrm{~nm} / \mathrm{nm}$ (Fig. 2). Closer to DTP this shift is 2.8 times higher $(0.216 \mathrm{~nm} / \mathrm{nm})$. It is necessary to precisely control the cladding reduction near to DTP. Too long etching process will cause complete annihilation of the resonance peaks.

Numerical simulations have shown that for the investigated grating the cladding radius reduction should reach $1.7 \mu \mathrm{m}$. Comparing the result with estimated by ellipsometric measurements $\mathrm{SiO}_{2}$ etching rate in $\mathrm{HF}$ buffer $(100 \mathrm{~nm} / \mathrm{min})$, the DTP should be reached in $17 \mathrm{~min}$ long etching process. In the experiment etching was interrupted several times in order to control resonance peak position. Amount of etching solution was as little as about $5 \mathrm{ml}$. Both of these aspects caused extension of etching process up to $18 \mathrm{~min}$ in order to etch comparably to simulated value amount of the cladding material. Comparison of simulated characteristics and etched LPG spectrum is shown in Fig. 3.

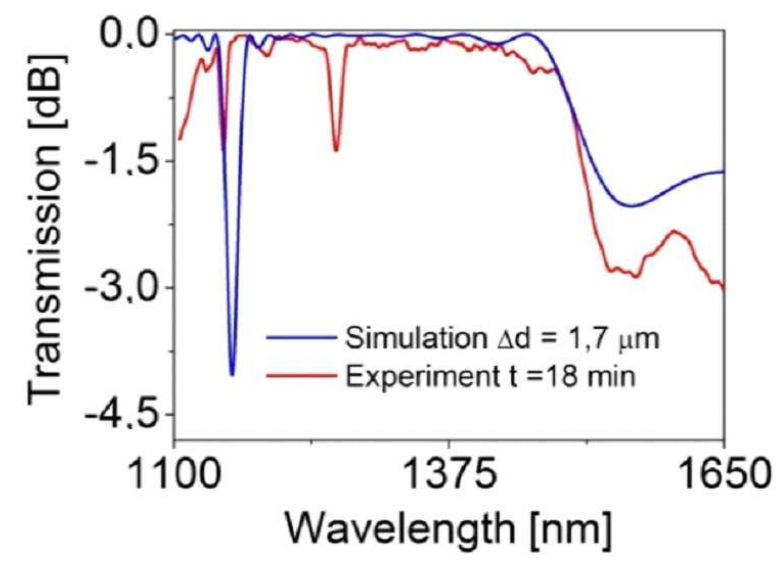

Fig. 3. Transmission spectrum simulation for $1.7 \mu \mathrm{m}$ cladding radius reduction compared to LPG etched in $18 \mathrm{~min}$ long process.

\subsection{RI sensitivity measurements}

Measurements of RI sensitivity were performed before and after cladding radius reduction. Sensitivity was calculated from linear fit as described in Sect. 2.4. Obtained RI sensitivity for non-etched LPG reaches SRI = $-190 \mathrm{~nm} / \mathrm{RIU}$. When the etching process was over, the measurements were performed again and sensitivity was over 5 times higher (SRI $=-971 \mathrm{~nm} / \mathrm{RIU}$ ) (Fig. 4).
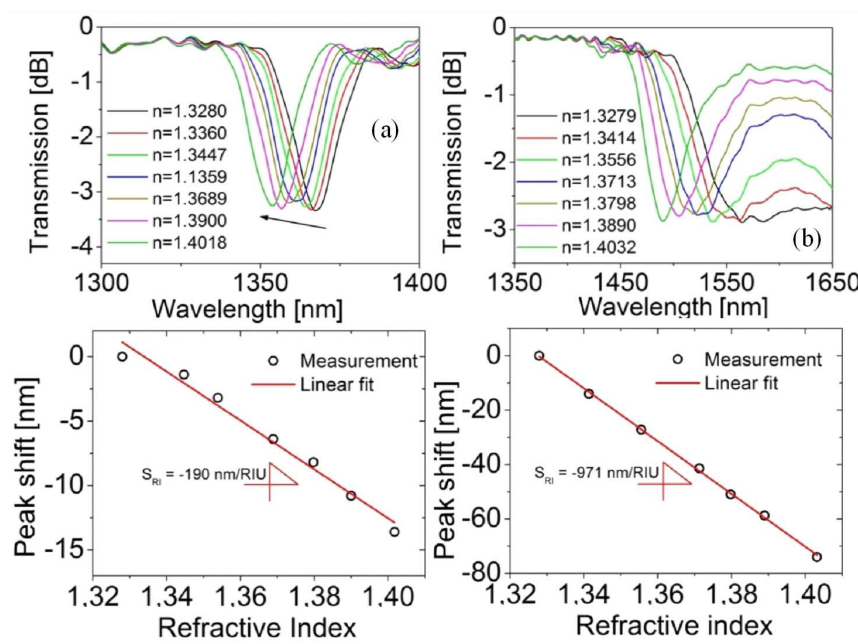

Fig. 4. Refractive index sensitivity, where (a) shows results before cladding radius reduction and (b) after etching-based post-processing procedure. Upper and lower graphs show transmission spectrum and resonant peak shift with change of RI, respectively.

\section{Conclusions}

In this work we successfully developed LPG sensor model using software package Optigrating by Optiwave. This model allows to quickly estimate location of DTP, as well as required conditions to achieve DTP. Simulation results show that cladding radius reduction enables 
achievement of maximum grating sensitivity. Thermally stable LPGs are fabricated with electric-arc technique, but physical limitations of this method does not allow periods shorter than $\approx 250 \mu \mathrm{m}$. Our experimental procedure shows that it is possible to apply post-fabrication etching to tune arc induced LPG to its maximum sensitivity point. $\mathrm{LPG}$ with period $\Lambda=283 \mu \mathrm{m}$ was etched in $\mathrm{HF}$ buffer for $\approx 18$ min which resulted in $\approx 1.7 \mu \mathrm{m}$ reduction of fibre cladding radius. This post-fabrication technique was successfully used to improve of RI sensitivity of LPG by a factor of 5 .

\section{Acknowledgments}

The authors gratefully acknowledge support for this work from the Ministry of Science and Higher Education of Poland within the Iuventus program, and the European Union in the framework of the Innovative Economy Operational Program through the Foundation for Polish Science Homing Plus Program.

\section{References}

[1] V. Bhatia, Opt. Expr. 4, 457 (1999).
[2] A.M. Vengsarkar, P.J. Lemaire, J.B. Judkins, V. Bhatia, T. Erdogan, J.E. Sipe, J. Lightwave Technol. 14, 58 (1996).

[3] K.O. Hill, G. Meltz, J. Lightwave Technol. 15, 1263 (1997).

[4] S. Xuewen, Z. Lin, I. Bennion, J. Lightwave Technol. 20, 255 (2002).

[5] Y. Wang, J. Appl. Phys. 108, 081101 (2010).

[6] T. Allsop, K. Kalli, K. Zhou, Y. Lai, G. Smith, M. Dubov, D.J. Webb, I. Bennion, Opt. Commun. 281, 5092 (2008).

[7] G. Rego, J. Opt. 12, 1 (2010).

[8] S. Park, O.-J. Kwon, Y.-G. Han, in: Communications and Photonics Conf. and Exhibition (ACP), Shanghai 2010, p. 94.

[9] J.M. Lazaro, A. Quintela, J.M. Lopez-Higuera, Sensors (Basel) 9, 5649 (2009).

[10] M. Smietana, W.J. Bock, P. Mikulic, J. Chen, Meas. Sci. Technol. 22, 015201 (2011).

[11] K. Zhou, H. Liu, X. Hu, Opt. Commun. 197, 295 (2001).

[12] W.J. Bock, J. Chen, P. Mikulic, T. Eftimov, IEEE Trans. Instrum. Meas. 56, 1176 (2007). 